\title{
The signs of ocular-surface disorders after switching from latanoprost to tafluprost/timolol fixed combination: a prospective study
}

This article was published in the following Dove Press journal:

Clinical Ophthalmology

21 June 2017

Number of times this article has been viewed

\author{
Hideaki Okumichi' \\ Yoshiaki Kiuchi' \\ Tetsuya Baba \\ Takashi Kanamoto ${ }^{3}$ \\ Tomoko Naito ${ }^{4,5}$ \\ Shunsuke Nakakura ${ }^{6}$ \\ Hitoshi Tabuchi ${ }^{6}$ \\ Hiroki $\mathrm{Nii}^{7}$ \\ Chie Sueoka ${ }^{7}$ \\ Yosuke Sugimoto ${ }^{1,8}$ \\ 'Department of Ophthalmology and \\ Visual Science, Graduate School \\ of Biomedical \& Health Sciences, \\ Hiroshima University, Hiroshima, \\ Japan; ${ }^{2}$ Shirai Eye Hospital, Mitoyo, \\ Japan; ${ }^{3}$ Department of Ophthalmology, \\ Hiroshima Memorial Hospital, \\ Hiroshima, Japan; ${ }^{4}$ Department of \\ Ophthalmology, Okayama University \\ Graduate School of Medicine, \\ Okayama, Japan; ${ }^{5}$ Department of \\ Ophthalmology, Konko Hospital, \\ Asakuchi, Japan; ${ }^{6}$ Department of \\ Ophthalmology, Saneikai Tsukazaki \\ Hospital, Himeji, Japan; ${ }^{7}$ Department \\ of Ophthalmology, Hiroshima \\ General Hospital, Hiroshima, Japan; \\ ${ }^{8}$ Department of Ophthalmology, \\ Hiroshima Prefectural Hospital, \\ Hiroshima, Japan
}

Correspondence: Yoshiaki Kiuchi Department of Ophthalmology and Visual Science, Graduate School of Biomedical \& Health Sciences, Hiroshima University, I-2-3 Kasumi, Minamiku, Hiroshima 734855I, Japan

$\mathrm{Tel}+8 \mid \mathrm{I} 822575247$

Fax +8I 822575249

Email ykiuchi@hiroshima-u.ac.jp
Purpose: To evaluate the ocular-surface safety of a $0.001 \%$ benzalkonium chloride-containing tafluprost/timolol fixed combination (TTFC) in patients with primary open-angle glaucoma (POAG) or ocular hypertension who have inadequate intraocular pressure (IOP) control with latanoprost monotherapy.

Methods: This study is a multicenter, prospective, single-arm, open-label clinical study. Patients with POAG or ocular hypertension who have inadequate IOP control with latanoprost monotherapy were considered eligible. After providing informed consent, patients continued latanoprost monotherapy for 12 weeks, followed by a switch to TTFC. We evaluated the extent of ocular-surface damage using superficial punctate keratopathy (SPK) score, tear breakup time (TBUT), hyperemia score, IOP, systolic blood pressure (SBP), diastolic blood pressure (DBP), and heart rate at 0,4 , and 12 weeks after switching.

Results: A total of 68 patients were enrolled, of whom, 64 patients were included in the final analysis. No significant changes in SPK score, TBUT, or hyperemia score were observed at 4 and 12 weeks compared with week 0 . IOP decreased significantly at $4(13.9 \pm 2.5 \mathrm{mmHg})$ and $12(14.1 \pm 2.5 \mathrm{mmHg})$ weeks, relative to week $0(15.3 \pm 2.7 \mathrm{mmHg})$. No significant changes in either SBP or DBP were observed during the study, although patients' mean heart rate decreased significantly after switching to TTFC. Adverse drug reactions to TTFC occurred in seven patients including two incidences of asthma and one of arrhythmia, and no events were serious.

Conclusion: The ocular-surface safety of TTFC is not significantly different to that of latanoprost. Furthermore, switching from latanoprost to TTFC in patients with insufficient IOP control has additive IOP-lowering effects. TTFC is an effective approach for patients receiving latanoprost monotherapy who require more intensive IOP reduction.

Keywords: tafluprost/timolol fixed combination, latanoprost, glaucoma, ocular-surface safety, intraocular pressure

\section{Introduction}

Glaucoma is a progressive neurodegenerative disease affecting the optic nerve and is a major cause of blindness. ${ }^{1,2}$ Reduction of intraocular pressure (IOP) is currently the only evidence-based treatment of glaucoma. The initial treatment approach for patients with primary open-angle glaucoma (POAG), the commonest type of glaucoma, is usually to reduce IOP using a single topical ocular hypotensive agent, such as a prostaglandin analog. Prostaglandin analogs are highly effective IOP-lowering agents, although combination treatment with two or more IOP-lowering agents is required for adequate long-term control of IOP in almost half of all patients with POAG. ${ }^{3}$ However, the concurrent use of multiple IOP-lowering agents often leads to problems such as poor 
adherence to treatment and ocular-surface disorders (OSDs). Fixed combinations have the advantage of better adherence compared with treatment with separate formulations of the same component medications. ${ }^{4}$ Patients with POAG require continuous treatment, owing to the fact that IOP-lowering agents are non-curative and can only prevent or delay disease progression. To encourage good adherence to treatment, IOPlowering agents should be well tolerated and safe because adverse effects increase the risk of poor adherence. Therefore, physicians should aim to prescribe fixed combinations of IOP-lowering agents to patients with glaucoma.

Tafluprost/timolol fixed combination (TTFC) is a commercially available formulation (Tapcom ${ }^{\circledR}$, Santen Pharmaceutical Co., Ltd., Osaka, Japan) consisting of a fixed-dose combination of a prostaglandin analog (tafluprost) and a $\beta$-blocker (timolol). TTFC has been previously shown to significantly reduce IOP after switching from tafluprost monotherapy (Tapros ${ }^{\circledR}$, Santen Pharmaceutical Co., Ltd.). ${ }^{5,6}$

The long-term use of multiple topical IOP-lowering agents may cause OSDs, and such disorders are much more prevalent in patients with $\mathrm{POAG}$ than in the general population. ${ }^{7,8}$ OSDs can be caused by chronic exposure to IOP-lowering medications and preservatives. ${ }^{9}$ Benzalkonium chloride (BAC) is a typical preservative used in ophthalmic solutions, and, at high concentrations, can cause keratoconjunctival epitheliopathy. ${ }^{9}$ Owing to the risk of toxic effects with long-term use, preservative-free or less toxic formulations of IOP-lowering agents have been developed to reduce the risk of OSDs. Treatment with tafluprost formulations containing $0.001 \% \mathrm{BAC}$ has been shown to result in a lower prevalence of superficial punctate keratopathy (SPK) than treatment with standard latanoprost formulations containing $0.02 \%$ BAC (Xalatan ${ }^{\circledR}$, Pfizer Inc., New York, NY, USA). ${ }^{10}$ In addition, certain active ingredients such as timolol might also encourage the development of corneal epithelial disorders. ${ }^{11}$ TTFC contains $0.001 \%$ BAC, less than many other formulations and, similar to tafluprost, results in lower levels of corneal cytotoxicity; however, this formulation also contains timolol, which may increase the risk of corneal cytotoxicity. However, the exact safety profile of TTFC in clinical use remains unknown.

In this study, we investigated the ocular-surface safety and IOP-lowering effects of TTFC after switching from latanoprost monotherapy. We evaluated ocular-surface safety by measuring SPK score, tear breakup time (TBUT), and hyperemia score. We also evaluated the systemic effects of TTFC, which are a major safety concern, by measuring systolic blood pressure (SBP), diastolic blood pressure (DBP) and heart rate, and the occurrence of adverse drug reactions using clinical observation and medical interviews.

\section{Methods \\ Study design}

This study is a multicenter, prospective, single-arm, open-label clinical study, registered as UMIN000016465 in the University Hospital Medical Information Network Clinical Trials Registry (UMIN-CTR). Patients were enrolled at one of seven participating hospitals, and the study protocol was approved by the Institutional Review Boards of Hiroshima University Hospital, Shirai Hospital, Hiroshima Memorial Hospital, Konko Hospital, Saneikai Tsukazaki Hospital, Hiroshima General Hospital, and Hiroshima Prefectural Hospital. The study design is in compliance with the Tenets of The Declaration of Helsinki and Ethical Guidelines for Clinical Research of the Japanese Ministry of Health, Labour and Welfare.

\section{Patients}

Patients with POAG or ocular hypertension were included in this study, inclusion criteria included age over 20 years, POAG or ocular hypertension of unilateral or bilateral eye(s), insufficient IOP control, but good adherence with latanoprost monotherapy $\left(\right.$ Xalatan $\left.^{\circledR}\right)$. Patients receiving treatment for keratoconjunctival epithelial disorder or dry eye, patients who had received corneal refractive surgery, those using contact lenses, those with active inflammatory or infectious diseases of the eye or eyelid, those who had undergone ocular surface or intraocular surgery within 12 months or glaucoma surgery including laser surgery within 12 months, patients who were contraindicated to $\beta$-blockers, or who were hypersensitive to any ingredients of latanoprost or TTFC, or were pregnant or planned pregnancy for the study period, breast feeding, or failed to meet any one of the inclusion criteria were excluded from this study.

Patients were enrolled between January 2015 and March 2016, after informed written consent was provided. Each patient initially continued to receive latanoprost (preserved with $0.02 \%$ BAC) monotherapy, applied once daily in the morning for 12 weeks as the run-in period (latanoprost phase), followed by a switch to TTFC (preserved with $0.001 \%$ BAC) applied once daily in the morning for 12 weeks (TTFC phase). The end of the latanoprost phase was defined as "week 0 ". All study participants underwent ocular and systemic examinations at $-12,-8,0,4$, and 12 weeks (Figure 1). No IOP-lowering medications other than latanoprost or TTFC nor ocular topical medications, including drugs to treat dry eye or keratoconjunctival disorders were allowed during the study period.

If both eyes satisfied the study criteria, we selected the study eye on the basis of higher SPK score, higher IOP, or if both of these parameters were equal, the right eye. 


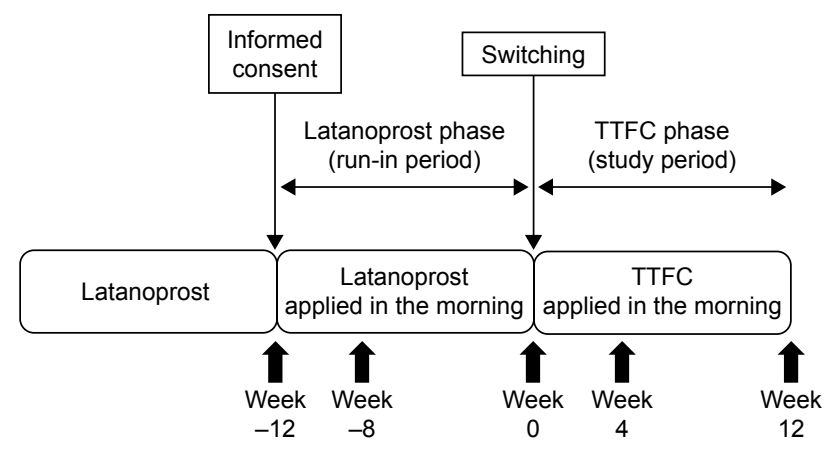

Figure I Study design.

Abbreviation: TTFC, tafluprost/timolol fixed combination.

\section{Measurements}

Slit-lamp examinations with fluorescein staining were used to examine patients' eyes prior to SPK scoring and measurement of TBUT. We graded the presence of SPK in three areas of the cornea (superior, central, inferior) separately between 0 and 3 (Table 1) and the total SPK score was calculated. TBUT was measured three times and the average was determined. We graded the presence of conjunctival hyperemia between 0 and 3 (Table 1). SPK scores and hyperemia scores were graded using standard-grading photographs. ${ }^{12}$ IOP was measured three times using a Goldmann applanation tonometer at as close to the same time of day during every visit as possible, and the average value was determined.

To examine systemic effects, SBP, DBP, and heart rate were measured. If an adverse event was detected, the physician evaluated the possibility of a causal relationship with the drug. Events for which a causal relationship with use of the drug could not be excluded were classified as adverse drug reactions.

\section{Statistical analyses}

Intragroup differences in SPK scores and hyperemia scores were analyzed using a Wilcoxon's signed-rank test. TBUT, IOP, SBP, DBP, and heart rate were analyzed using a paired $t$-test. Data were analyzed using R statistical analysis software version 3.3.1 (64 bit [2016], The R Foundation for Statistical Computing, Vienna, Austria, http://www.R-project.org/). All $P$-values were two-sided and were considered statistically

Table I Grading of SPK score and hyperemia score

\begin{tabular}{lll}
\hline & SPK score & Hyperemia score \\
\hline 0 & No staining & No hyperemia \\
I & Partial staining of less than half of the area & Slight hyperemia \\
2 & Staining of more than half of the area & Moderate hyperemia \\
3 & Staining of the entire area & Severe hyperemia \\
\hline
\end{tabular}

Notes: Data from Kanamoto et al. ${ }^{12}$ SPK score was evaluated separately in three sections (the superior area, the central area, and the inferior area).

Abbreviation: SPK, superficial punctate keratopathy. significant when the values were less than 0.05. If the patient used TTFC in both eyes, and the IOP of the study eye was lower than that of the other eye at week 0 , we excluded the patient from IOP analysis.

Data were collected by Bell Medical Solutions, Inc., Tokyo, Japan. All statistical analyses were conducted by H.T. of Saneikai Tsukazaki Hospital, Himeji, Japan, all analyses were inspected by Sugimoto Data Analysis Service.

\section{Sample size}

We estimated that 70 patients would be required to detect a mean difference of 0.3 in SPK score between week 0 and week 12 , with a SD of $0.88,{ }^{12}$ at a two-sided $\alpha$ level of $5 \%$ and a power of $80 \%$. Considering the possibility of discontinuation of treatment and dropout from the study cohort, we planned to enroll 85 patients at the beginning of this study. Interim results from 50 patients who completed the study by March 2016 showed that it was possible to evaluate the ocular-surface safety of TTFC. Further patient enrollment was terminated at that time.

\section{Results}

Sixty-eight patients participated in this study and two withdrew during the latanoprost phase. The remaining 66 patients (37 men and 29 women) received TTFC treatment. Mean age upon enrollment $( \pm \mathrm{SD})$ was $68.2 \pm 11.5$ years. Sixtyfour patients had POAG and two had ocular hypertension. One patient discontinued TTFC immediately after switching owing to an adverse reaction to TTFC, and another patient, who was suspected of having exfoliation glaucoma, was disqualified from the study. Following exclusion of these two patients, 64 patients were included in the final analysis.

Patients' mean SPK scores in all areas (the superior area, the central area, the inferior area, and also the total area) at weeks 4 and 12 were not significantly different to those obtained at week 0 (Table 2). Forty-four patients (69\%) had a total SPK score of 0,10 patients $(16 \%)$ had a total SPK score of 1, eight patients (13\%) had a total SPK score of 2,

Table 2 SPK score

\begin{tabular}{|c|c|c|c|c|c|}
\hline \multirow[t]{2}{*}{ Area } & \multirow{2}{*}{$\frac{\text { Week } 0}{\text { SPK score }}$} & \multicolumn{2}{|l|}{ Week 4} & \multicolumn{2}{|l|}{ Week I2 } \\
\hline & & SPK score & $P$-value & SPK score & $P$-value \\
\hline Superior & $0.02 \pm 0.13$ & $0.00 \pm 0.00$ & 1.00 & $0.02 \pm 0.13$ & 1.00 \\
\hline Central & $0.17 \pm 0.38$ & $0.08 \pm 0.27$ & 0.27 & $0.06 \pm 0.24$ & 0.07 \\
\hline Inferior & $0.3 \mathrm{I} \pm 0.50$ & $0.24 \pm 0.43$ & 0.39 & $0.33 \pm 0.54$ & 1.00 \\
\hline Total area & $0.50 \pm 0.84$ & $0.32 \pm 0.57$ & 0.07 & $0.4 I \pm 0.68$ & 0.43 \\
\hline
\end{tabular}

Notes: Data are presented as mean \pm SD. All $P$-values indicate the results of comparisons with the same values obtained at week 0 . No statistically significant differences were observed (Wilcoxon signed-rank test).

Abbreviation: SPK, superficial punctate keratopathy. 


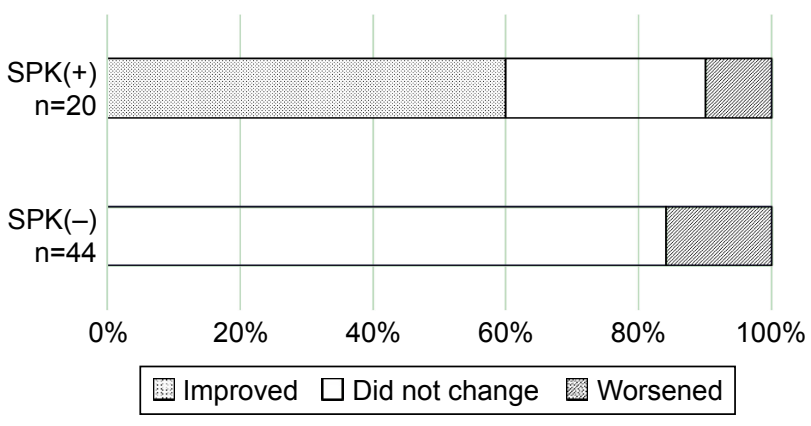

Figure 2 Changes in SPK score.

Notes: Of the 20 patients who had an SPK at week 0, 12 patients $(60 \%)$ had an improved SPK score at week 12, six patients (30\%) had no change in SPK score, and two patients (10\%) had a worse SPK score. Of the 44 patients without an SPK score at week 0 , seven patients (16\%) developed an SPK score at week 12.

Abbreviation: SPK, superficial punctate keratopathy.

and two patients $(3 \%)$ had a total SPK score of 3 . Of the 20 patients with a total SPK score of 1-3 at week 0 , this score decreased in 12 patients $(60 \%)$ after 12 weeks, increased in two patients $(10 \%)$, and did not change in six patients $(30 \%)$. However, of the 44 patients without an SPK score at week 0, seven patients $(16 \%)$ had an increase in SPK score, indicating worsening of symptoms at week 12 (Figure 2).

Mean TBUTs at 0,4 , and 12 weeks were $6.8 \pm 3.6 \mathrm{~s}$, $7.0 \pm 3.4 \mathrm{~s}$ and $6.8 \pm 3.1 \mathrm{~s}$, respectively. No statistically significant differences in TBUT were observed at 4 and 12 weeks (paired $t$-test, $P=0.78, P=0.95$,) compared with TBUT at week 0 (Figure 3).

Hyperemia scores at 0,4 , and 12 weeks were $0.36 \pm 0.52$, $0.29 \pm 0.49$ and $0.27 \pm 0.45$, respectively. No statistically significant differences in hyperemia scores were observed at 4 and 12 weeks (Wilcoxon's signed rank test, $P=0.55$, $P=0.18$ ) compared with week 0 (Figure 4).

IOP levels of 57 patients were evaluated (56 patients at weeks 4 and 12, owing to lack of data from one patient). IOP at weeks $-12,0,4$, and 12 was $15.2 \pm 2.5 \mathrm{mmHg}, 15.3 \pm 2.7 \mathrm{mmHg}$,

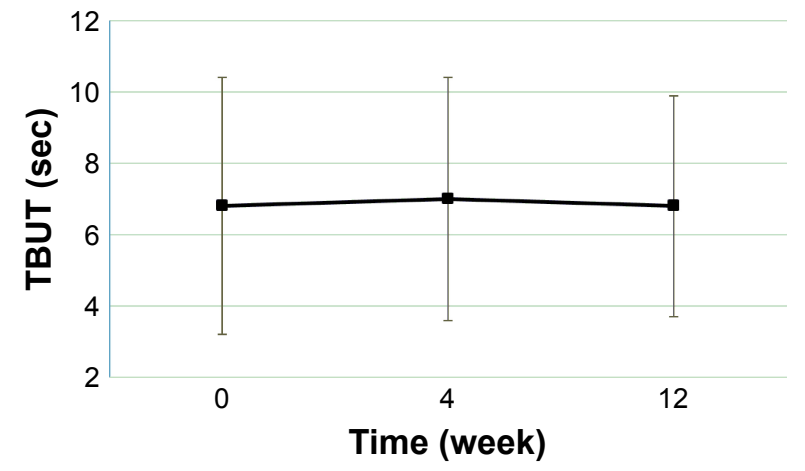

Figure 3 TBUT.

Notes: Data are presented as mean \pm SD. No statistically significant differences were observed at weeks 4 and 12 (paired $t$-test; $P=0.78, P=0.95$ ) compared with week 0 . Abbreviation: TBUT, tear breakup time.

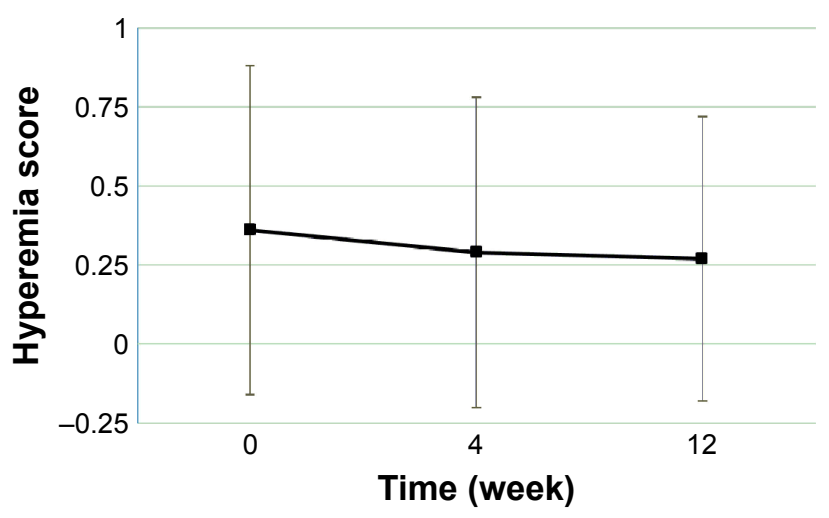

Figure 4 Hyperemia score.

Notes: Data are presented as mean \pm SD. No statistically significant differences were observed at weeks 4 and 12 (Wilcoxon signed-rank test; $P=0.55, P=0.18$ ) compared with week 0 .

$13.9 \pm 2.5 \mathrm{mmHg}$, and $14.1 \pm 2.5 \mathrm{mmHg}$, respectively. No statistically significant differences between IOP levels were observed at weeks -12 and 0 (paired $t$-test, $P=0.64$ ); however, significant decreases in IOP levels were observed at weeks 4 and 12 (paired $t$-test, $P<0.0001$,) compared with week 0 (Figure 5).

Mean SBP at weeks 0, 4, and 12 was $127.9 \pm 17.5 \mathrm{mmHg}$, $125.5 \pm 18.9 \mathrm{mmHg}$, and $129.9 \pm 19.0 \mathrm{mmHg}$, respectively. No statistically significant changes in SBP were observed at weeks 4 and 12 (paired $t$-test, $P=0.13, P=0.36$, respectively) compared with week 0. Similarly, mean DBP at weeks 0, 4 , and 12 was $73.0 \pm 10.9 \mathrm{mmHg}, 73.0 \pm 10.7 \mathrm{mmHg}$, and $73.8 \pm 11.9 \mathrm{mmHg}$, respectively, with no statistically significant differences at weeks 4 and 12 (paired $t$-test, $P=0.82, P=0.61$, respectively) compared with week 0 (Figure 6).

Mean heart rate at weeks 0,4 , and 12 was $74.7 \pm 12.8 \mathrm{bpm}$, $69.7 \pm 10.7 \mathrm{bpm}$, and $68.7 \pm 11.3 \mathrm{bpm}$, respectively, reflecting a significant decrease at weeks 4 and 12 (paired $t$-test, $P<0.001, P<0.0001$,) compared with week 0 (Figure 7).

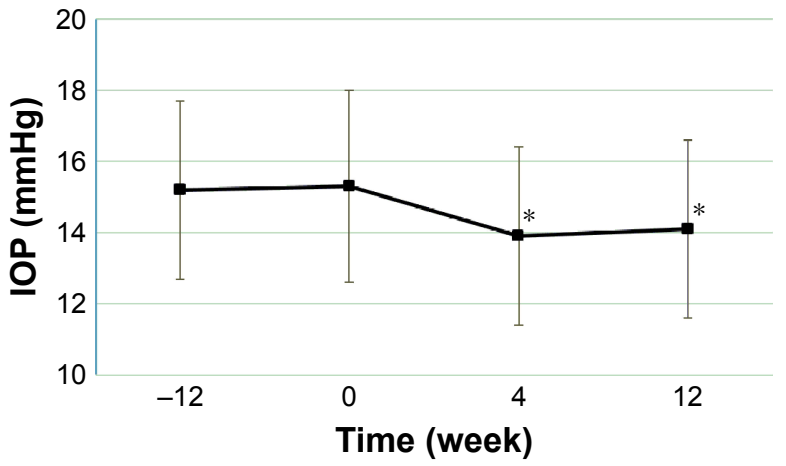

Figure 5 IOP.

Notes: Data are presented as mean \pm SD. *Indicates $P<0.05$, paired $t$-test (vs week 0 ). No statistically significant differences were observed between weeks -12 and 0 $(P=0.64$, paired $t$-test). IOP decreased significantly at weeks 4 and 12 (paired $t$-test; $P<0.000$ I) compared with week 0 .

Abbreviation: IOP, intraocular pressure. 


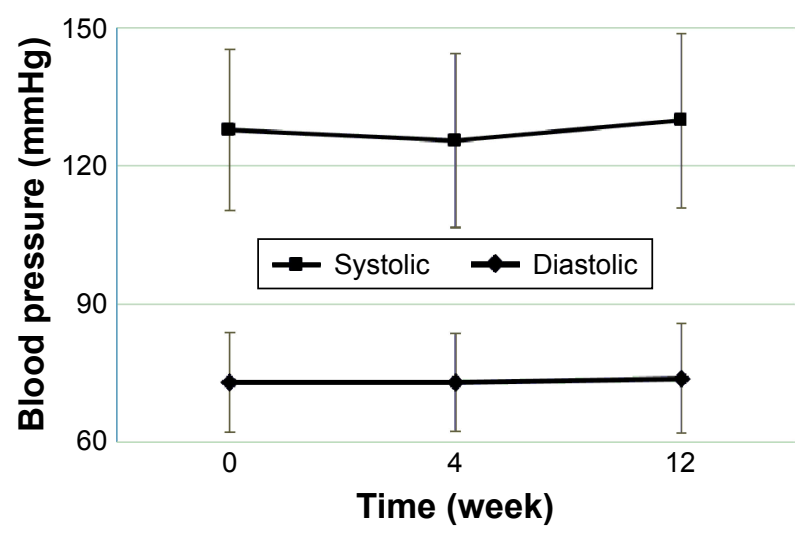

Figure 6 Blood pressure.

Notes: Data are presented as mean \pm SD. No statistically significant differences in systolic or diastolic blood pressure were observed at weeks 4 (paired $t$-test; $P=0.13$, $P=0.82$ ) and 12 (paired $t$-test; $P=0.36, P=0.61$ ) compared with week 0 .

Adverse reactions to TTFC occurred in seven patients; self-reported asthma in two patients, and arrhythmia, blepharitis, shoulder stiffness, eye irritation and eye pain in one patient each. All of the observed adverse events were considered non-serious and patients recovered with/without discontinuation of treatment (Table 3 ).

\section{Discussion}

Reduction of IOP is the first priority of glaucoma treatment. In this study, patients with POAG or ocular hypertension who had insufficient control of elevated IOP on latanoprost monotherapy were switched to TTFC. To eliminate the possibility that the true IOP-lowering effects of TTFC are simply the result of improved adherence owing to study participation, the run-in period was set to 12 weeks. During this period, patients continued to use latanoprost. As a result, no statistically significant differences in IOP were observed between the beginning (week -12) and the end (week 0) of the latanoprost phase. After switching to TTFC, a significant

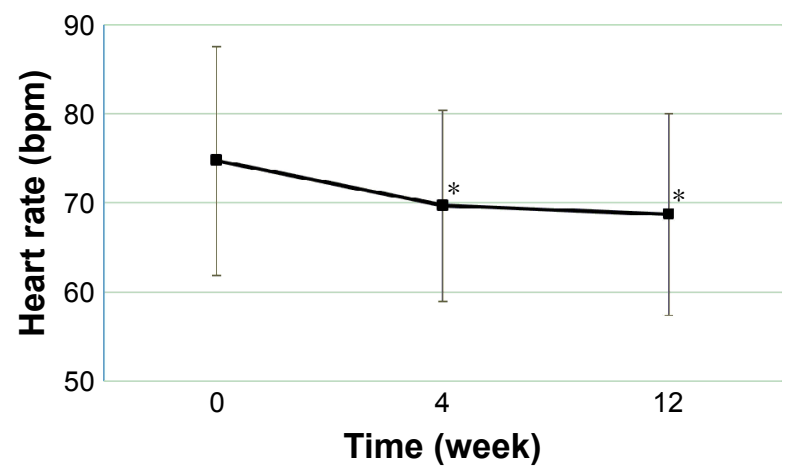

Figure 7 Heart rate.

Notes: Data are presented as mean \pm SD. *Indicates $P<0.05$, paired $t$-test (vs week 0 ). A significant decrease in heart rate was observed at weeks 4 and $I 2$ (paired $t$-test; $P<0.00$ I, $P<0.000$ I) compared with week 0 .
Table 3 Adverse drug reactions

\begin{tabular}{lcl}
\hline & & Instillation \\
\hline $\begin{array}{l}\text { Latanoprost phase } \\
\text { Eye discharge }\end{array}$ & $\mathrm{I}$ & Continued \\
$\begin{array}{l}\text { TTFC phase } \\
\text { Asthma }\end{array}$ & 2 & Discontinued \\
Arrhythmia & $\mathrm{I}$ & Discontinued \\
Blepharitis & $\mathrm{I}$ & Discontinued \\
Shoulder stiffness & $\mathrm{I}$ & Continued \\
Eye irritation & $\mathrm{I}$ & Continued \\
Eye pain & $\mathrm{I}$ & Continued \\
\hline
\end{tabular}

Abbreviation: TTFC, tafluprost/timolol fixed combination.

decrease in IOP was observed at weeks 4 and 12 compared with week 0 , as reported previously. ${ }^{5,6,13}$ These findings suggest that the IOP reduction observed in this study is an additive effect of the $\beta$-blocker, timolol, and not a result of improved adherence.

Keratoepithelial disorders caused by eye drops are mostly associated with the concentration of BAC in the formulation. Data from in vitro studies using human corneal epithelial cells and in vivo studies using rabbit models have revealed that BAC is the major cause of keratoepithelial disorders. ${ }^{14}$ After switching from latanoprost to a tafluprost formulation containing lower levels of BAC or to a travoprost formulation containing SofZia instead of BAC, the prevalence of SPK was found to decrease in two clinical studies. ${ }^{10,15}$ In addition, topical $\beta$-blockers, which decrease corneal sensitivity ${ }^{16}$ and the number of goblet cells in the conjunctiva, ${ }^{17}$ have also been associated with an increased risk of keratoepithelial disorders. ${ }^{11}$ The cytotoxic effects of timolol were revealed by data from an in vitro study comparing latanoprost with a fixed-combination latanoprost/timolol formulation containing $\mathrm{BAC}$ at the same concentration. In the presence of the same concentration of BAC, the latanoprost/timolol combination was more toxic to human corneal epithelial cells in vitro than latanoprost alone. ${ }^{18}$ In a comparison of two fixed combined formulations, TTFC had fewer cytotoxic effects on corneal epithelial cells in vitro than latanoprost/timolol, owing to a lower concentration of BAC. ${ }^{19}$ Our results indicate no statistically significant change in SPK score after switching from latanoprost to TTFC. Furthermore, no significant change in SPK score was observed after switching from latanoprost to travoprost/timolol without BAC. ${ }^{20}$ These findings suggest that the deleterious effects of timolol may counter the reduction in cytotoxicity owing to reduced exposure to BAC.

In this study, 69\% (44/64) of patients had no SPK at week 0 and the mean total SPK score was 0.50 . At 12 weeks of treatment, $60 \%(12 / 20)$ of the patients with an SPK score at week 0 had an improved SPK score, despite receiving no treatment for OSDs; however, SPK appeared in 16\% (7/44) 
of patients without an SPK score at week 0. The presence of patients with low SPK scores at week 0 might explain the finding of no statistically significant change in SPK scores between weeks 0 and 12, despite the majority of patients with an SPK score having an improvement in this parameter. A further study, in which only patients with high SPK scores are eligible for inclusion, may provide further insight.

Abnormal tear film conditions have been suggested to contribute to the development of OSDs. Anti-glaucoma eye drops containing BAC and $\beta$-blockers may also disturb the stability of tear films and affect TBUT. In previous studies, switching from prostaglandin formulations also containing high concentrations of BAC to tafluprost, containing lower concentrations of BAC led to the prolongation of TBUT, ${ }^{21}$ and the use of topical $\beta$-blockers shortened the duration of TBUT. ${ }^{22}$ In our study, TBUT was unaffected by switching from latanoprost to TTFC. The effects of BAC and $\beta$-blockers on TBUT might, therefore, cancel each other out, resulting in no overall change in TBUT.

Conjunctival hyperemia, which is the most frequent side effect of prostaglandins, ${ }^{23}$ causes concern to patients for cosmetic reasons and might also reduce their adherence to treatment. In a study using quantitative hyperemia analysis software, tafluprost, among the prostaglandin analogs tested, was reported to cause the lowest level of hyperemia. ${ }^{24}$ Data from a meta analysis ${ }^{25}$ indicate that receiving treatment with prostaglandin/timolol fixed combinations results in a lower risk of hyperemia than treatment with prostaglandins alone, although the mechanism of this effect is unknown. ${ }^{26}$ In this study, a reduction in hyperemia was expected after switching from latanoprost to TTFC, however, no significant differences in hyperemia scores were detected.

The potential for adverse effects on cardiovascular and respiratory systems is the major concern regarding the use of fixed combinations containing $\beta$-blockers. To evaluate the effects of timolol on cardiovascular function, SBP, DBP, and heart rate were measured in all patients. SBP and DBP did not change significantly, and heart rate decreased significantly after switching from latanoprost to TTFC. The same results were shown in previous studies involving switching from latanoprost to fixed combinations of travoprost/timolol ${ }^{20}$ and from tafluprost to TTFC. ${ }^{5}$ This reduction in heart rate might be induced by timolol, a non-selective $\beta$-adrenergic antagonist, ${ }^{27}$ although in this study, this reduction did not lead to clinical problems such as dyspnea or difficulties in daily life.

Asthma is a contraindication to the use of $\beta$-blockers. At enrollment, patients were confirmed, by interview, not to have asthma, although two patients developed asthma after the switch to TTFC. Reportedly, only $10 \%$ of asthma patients had a prior diagnosis. ${ }^{28}$ Therefore, physicians should not only ask the patient whether they have asthma or not before starting $\beta$-blockers, but should also monitor the condition of patients during the course of treatment. All of the adverse drug reactions observed with use of TTFC, including asthma and arrhythmia, were not serious and patients recovered.

As limitations, in our study investigators were not masked to the details of the treatment and this was not a cross-over study. Although we used the standard grading photographs to evaluate SPK and conjunctival hyperemia scores, these methods are subjective, and therefore could be affected by investigator bias. Furthermore, the observation period was only 12 weeks after switching to TTFC and we did not evaluate the effects of switching to TTFC in patients with other types of glaucoma, such as angle-closure glaucoma and secondary glaucoma. Only patients with insufficient IOP control were enrolled and the definition of insufficient IOP control was subject to the judgement of the examining physician. This might cause some selection bias, but also reflects the general clinical situation in which switching of IOP-lowering agents takes place. Finally, even if a major proportion of patients with SPK had improved SPK scores after switching to TTFC, this result might have been more pronounced had the inclusion criteria also required signs/ symptoms of OSDs while receiving prior medication.

\section{Conclusion}

The ocular-surface safety of TTFC is not significantly different to that of latanoprost. Furthermore, switching from latanoprost to TTFC in patients with insufficient IOP control has additive IOP-lowering effects. Therefore, TTFC is an effective approach for patients receiving latanoprost monotherapy who require more intensive IOP reduction.

\section{Acknowledgments}

Joji Takenaka, Masahide Yanagi, Ken Kobayashi (Hiroshima University) and Takaaki Sasaki (Hiroshima Prefectural Hospital) provided and cared for study patients, and reviewed the manuscript. Masayo Hashimoto, Naomi Otsuka, Hiroaki Kurashima, Takeshi Hizume, and Etsuyo Miyamoto (Department of Medical Affairs, Santen Pharmaceutical Co., Ltd., Osaka, Japan) provided assistance with study design and review of the manuscript. This study was sponsored and funded by Santen Pharmaceutical Co., Ltd., Osaka, Japan. The funding organization participated in the design of the study, interpretation of the data, review and approval of the 
manuscript and had no role in the conduct, data collection, or analysis of data derived from this study.

\section{Disclosure}

The authors report no conflicts of interest in this work.

\section{References}

1. Weinreb RN, Aung T, Medeiros FA. The pathophysiology and treatment of glaucoma: a review. JAMA. 2014;311(18):1901-1911.

2. Iwase A, Araie M, Tomidokoro A, Yamamoto T, Shimizu H, Kitazawa Y; Tajimi Study Group. Prevalence and causes of low vision and blindness in a Japanese adult population: the Tajimi Study. Ophthalmology. 2006;113(8):1354-1363

3. Kass MA, Heuer DK, Higginbotham EJ, et al. The Ocular Hypertension Treatment Study: a randomized trial determines that topical ocular hypotensive medication delays or prevents the onset of primary openangle glaucoma. Arch Ophthalmol. 2002;120(6):701-713.

4. Higginbotham EJ. Considerations in glaucoma therapy: fixed combinations versus their component medications. Clin Ophthalmol. 2010;4:1-9.

5. Kuwayama Y; DE-111 Collaborative Trial Group. [Phase III doublemasked study of fixed combination tafluprost $0.0015 \% /$ timolol $0.5 \%$ (DE-111) versus tafluprost $0.0015 \%$ alone or given concomitantly with timolol $0.5 \%$ in primary open angle glaucoma and ocular hypertension]. Atarashii Ganka. 2013;32(8):1185-1194. Japanese.

6. Kuwayama Y; DE-111 Collaborative Trial Group. [A long-term, openlabel study of fixed combination tafluprost $0.0015 \%$ /timolol $0.5 \%$ (DE-111) in patients with open-angle glaucoma or ocular hypertension] Atarashii Ganka. 2015;32(1):133-143. Japanese.

7. Ramli N, Supramaniam G, Samsudin A, Juana A, Zahari M, Choo MM. Ocular surface disease in glaucoma: effect of polypharmacy and preservatives. Optom Vis Sci. 2015;92(9):e222-e226.

8. Uzunosmanoglu E, Mocan MC, Kocabeyoglu S, Karakaya J, Irkec M. Meibomian gland dysfunction in patients receiving long-term glaucoma medications. Cornea. 2016;35(8):1112-1116.

9. Baudouin C, Labbé A, Liang H, Pauly A, Brignole-Baudouin F. Preservatives in eyedrops: the good, the bad and the ugly. Prog Retin Eye Res. 2010;29(4):312-334.

10. Kumagami T, Wakiyama H, Kusano M, et al. Comparison of corneal safety and intraocular pressure-lowering effect of tafluprost ophthalmic solution with other prostaglandin ophthalmic solutions. J Ocul Pharmacol Ther. 2014;30(4):340-345.

11. Lee S, Kim MK, Choi HJ, Wee WR, Kim DM. Comparative crosssectional analysis of the effects of topical antiglaucoma drugs on the ocular surface. Adv Ther. 2013;30(4):420-429.

12. Kanamoto T, Kiuchi Y, Tanito M, et al. Comparison of the toxicity profile of benzalkonium chloride-preserved tafluprost and SofZiapreserved travoprost applied to the ocular surface. J Ocul Pharmacol Ther. 2015;31(3):156-164.

13. Takagi Y, Osaki H, Yamashita T, Kai Y. Prospective observational post-marketing study of tafluprost $0.0015 \% /$ timolol $0.5 \%$ combination ophthalmic solution for glaucoma and ocular hypertension: short-term efficacy and safety. Ophthalmol Ther. 2016;5(2):191-206.

Clinical Ophthalmology

\section{Publish your work in this journal}

Clinical Ophthalmology is an international, peer-reviewed journal covering all subspecialties within ophthalmology. Key topics include: Optometry; Visual science; Pharmacology and drug therapy in eye diseases; Basic Sciences; Primary and Secondary eye care; Patient Safety and Quality of Care Improvements. This journal is indexed on Submit your manuscript here: http://www.dovepress.com/clinical-ophthalmology-journal
14. Pauly A, Roubeix C, Liang H, Brignole-Baudouin F, Baudouin C. In vitro and in vivo comparative toxicological study of a new preservative-free latanoprost formulation. Investig Ophthalmol Vis Sci. 2012;53(13): 8172-8180.

15. Aihara M, Oshima H, Araie M; EXTraKT study group. Effects of SofZia-preserved travoprost and benzalkonium chloride-preserved latanoprost on the ocular surface - a multicentre randomized singlemasked study. Acta Ophthalmol. 2013;91(1):e7-e14.

16. Weissman SS, Asbell PA. Effects of topical timolol $(0.5 \%)$ and betaxolol (0.5\%) on corneal sensitivity. Br J Ophthalmol. 1990;74(7):409-412.

17. Aydin Kurna S, Acikgoz S, Altun A, Ozbay N, Sengor T, Olcaysu OO. The effects of topical antiglaucoma drugs as monotherapy on the ocular surface: a prospective study. J Ophthalmol. 2014;2014:460483.

18. Nagai N, Murao T, Oe K, Ito Y, Okamoto N, Shimomura Y. [In vitro evaluation for corneal damages by anti-glaucoma combination eye drops using human corneal epithelial cell (HCE-T)]. Yakugaku Zasshi. 2011;131(6):985-991. Japanese.

19. Fuwa M, Ueda K, Akaishi T, et al. Advantages of efficacy and safety of fixed-dose tafluprost/timolol combination over fixed-dose latanoprost/ timolol combination. PLoS One. 2016;11(7):e0158797.

20. Kashiwagi K. Efficacy and safety of switching to travoprost/timolol fixed-combination therapy from latanoprost monotherapy. Jpn J Ophthalmol. 2012;56(4):339-345.

21. Suzuki K, Teranishi S, Sagara T, et al. Safety and efficacy of benzalkonium chloride-optimized tafluprost in Japanese glaucoma patients with existing superficial punctate keratitis. J Glaucoma. 2015; 24(6):e145-e150.

22. Arita R, Itoh $\mathrm{K}$, Maeda $\mathrm{S}$, et al. Effects of long-term topical antiglaucoma medications on meibomian glands. Graefes Arch Clin Exp Ophthalmol. 2012;250(8):1181-1185.

23. Zimmerman TJ, Hahn SR, Gelb L, Tan H, Kim EE. The impact of ocular adverse effects in patients treated with topical prostaglandin analogs: changes in prescription patterns and patient persistence. J Ocul Pharmacol Ther. 2009;25(2):145-152.

24. Yanagi M, Kiuchi Y, Yuasa Y, et al. Association between glaucoma eye drops and hyperemia. Jpn J Ophthalmol. 2016;60(2):72-77.

25. Quaranta L, Biagioli E, Riva I, et al. Prostaglandin analogs and timololfixed versus unfixed combinations or monotherapy for open-angle glaucoma: a systematic review and meta-analysis. J Ocul Pharmacol Ther. 2013;29(4):382-389.

26. Radcliffe NM. The impact of timolol maleate on the ocular tolerability of fixed-combination glaucoma therapies. Clin Ophthalmol. 2014; 8:2541-2549.

27. Dickstein K, Hapnes R, Aarsland T. Comparison of aqueous and gellan ophthalmic timolol with placebo on the 24-hour heart rate response in patients on treatment for glaucoma. Am J Ophthalmol. 2001; 132(5):626-632.

28. Fukuchi Y, Nishimura M, Ichinose M, et al. COPD in Japan: the Nippon COPD Epidemiology study. Respirology. 2004;9(4):458-465.

\section{Dovepress}

PubMed Central and CAS, and is the official journal of The Society of Clinical Ophthalmology (SCO). The manuscript management system is completely online and includes a very quick and fair peer-review system, which is all easy to use. Visit http://www.dovepress.com/ testimonials.php to read real quotes from published authors. 